\title{
Design and Experimental Implementation of a Beam-Type Twin Dynamic Vibration Absorber for a Cantilevered Flexible Structure Carrying an Unbalanced Rotor: Numerical and Experimental Observations
}

\author{
Abdullah Özer, ${ }^{1}$ Mojtaba Ghodsi, ${ }^{1}$ Akio Sekiguchi, ${ }^{2}$ \\ Ashraf Saleem, ${ }^{3}$ and Mohammed Nasser Al-Sabari ${ }^{1}$ \\ ${ }^{1}$ Department of Mechanical and Industrial Engineering, College of Engineering, Sultan Qaboos University, \\ P.O. Box 33, Al-Khoud, 123 Muscat, Oman \\ ${ }^{2}$ Department of Control Engineering, Kisarazu National College of Technology, 2 Chome-11-1 Kiyomidaihigashi, \\ Kisarazu, Chiba 292-0041, Japan \\ ${ }^{3}$ Department of Computer and Electrical Engineering, College of Engineering, Sultan Qaboos University, \\ P.O. Box 33, Al-Khoud, 123 Muscat, Oman
}

Correspondence should be addressed to Abdullah Özer; ozer@squ.edu.om

Received 8 April 2015; Accepted 30 July 2015

Academic Editor: Sergio De Rosa

Copyright (C) 2015 Abdullah Özer et al. This is an open access article distributed under the Creative Commons Attribution License, which permits unrestricted use, distribution, and reproduction in any medium, provided the original work is properly cited.

\begin{abstract}
This paper presents experimental and numerical results about the effectiveness of a beam-type twin dynamic vibration absorber for a cantilevered flexible structure carrying an unbalanced rotor. An experimental laboratory prototype setup has been built and implemented in our laboratory and numerical investigations have been performed through finite element analysis. The proposed system design consists of a primary cantilevered flexible structure with an attached dual-mass cantilevered secondary dynamic vibration absorber arrangement. In addition, an unbalanced rotor system is attached to the tip of the flexible cantilevered structure to inspect the system response under harmonic excitations. Numerical findings and experimental observations have revealed that significant vibration reductions are possible with the proposed dual-mass, cantilevered dynamic vibration absorber on a flexible cantilevered platform carrying an unbalanced rotor system at its tip. The proposed system is efficient and it can be practically tuned for variety of design and operating conditions. The designed setup and the results in this paper can serve for practicing engineers, researchers and can be used for educational purposes.
\end{abstract}

\section{Introduction}

When machines and flexible structures are subjected to a repeating type of external forces, vibrations inevitably occur in their components. Particularly, if the frequency of the external force coincides with one of the natural vibration frequencies of the system, a condition known as resonance occurs, and the system undergoes dangerously large oscillations [1-3]. Vibrations in structures and machines can lead to instability, fatigue, and eventually the failure of the structure which can be catastrophic in some cases. Failures of structures such as buildings, bridges, turbines, and airplane wings can be associated with the occurrence of resonance. Consequently, vibration analysis and vibration control have significant practical importance in design and operation of machines and flexible structures.

Many control techniques have been proposed to control the vibrations of flexible structures. The control methods for vibrations can be grouped into two broad categories: passive control and active control [4]. Passive control methods require an addition of secondary structure to the primary system, usually defined as auxiliary dynamic vibration absorber. A passive controller aims at designing the mass, stiffness, 
and energy dissipation properties of structures to minimize dynamic response. Usually, passive control techniques require design modifications and well tuning for changing operational conditions. Effectiveness of a passive vibration controller may deteriorate drastically for varying design and operational conditions; therefore they may have limited effectiveness for some vibration problems. Hence, designing a passive controller which is adoptable to varying conditions is an important task for the design engineer. On the other hand, passive vibration control techniques can be simple and sufficient solution in many applications. Consequently, active vibration control strategies have not been able to entirely replace the passive counterparts in spite of vast technological improvements in active control procedures and accurate measurement techniques. However, there could be cases where active vibration control techniques can be a favorable solution as compared to passive counterparts. Nevertheless, in cases where performance enhancements are very comparable, usually passive controllers are favored due to their simplicity and low costs.

One of the causes of unwanted vibrations could be rotating machinery which usually comprises unbalanced components. The unbalance is usually caused by the fact that the center of mass of the rotating part does not coincide with the center of rotation. In order to operate rotating machinery with increased productivity and better performance, it is required to design reliable systems to control the undesired vibrations.

Many control techniques have been proposed in recent decades to attenuate the undesired vibrations of flexible structures. Sophisticated actuators along with highly developed computer control and measurement procedures have greatly increased the number of applications for active control of vibrations. In active control, the sensor measures the motion of the system or flexible structure (either its displacement, velocity, or acceleration, depending on the application) and the control algorithm determines the required force or torque. Subsequently, this information is sent to the actuators as an input parameter and the actuators impose the required forces or torques online. Several researchers have worked in the area of active vibration control. In an earlier research, Okada et al. [5] introduced a noncontact method of active vibration control for reducing the flexural vibrations of a thin steel sheet. The proposed electromagnetic method of control has been implemented in a simple experimental setup where the signal from a motion sensor regulates the attractive force of the magnets that produce a damping force on the steel sheet. Recently, Biglar et al. [6, 7] investigated optimal locations and orientations of piezoelectric transducers patches in different case studies. To control vibrations, negative velocity feedback control and saturated negative velocity feedback rules are considered. It is shown that proposed methods increase the effectiveness and the vibrations are suppressed more quickly. Özer et al. applied active control strategies for flexible robot manipulators in different applications [4, 8-10]. Recently, Zhao and $\mathrm{Hu}$ [11] have investigated active vibration control of an axially translating robot arm with rotatingprismatic joint using self-sensing actuator. Their numerical simulations proved that the tip deflection of the arm can be effectively reduced by the proposed self-sensing actuator. One of the potential methods to deal with modeling and parameter changes is robust controller design, including robust pole-placement [12], robust $\mathrm{H}_{\infty}$ [13], robust LQ [14], and robust min-max LQ [15].

Many mathematical models of flexible structures have been developed for active or passive vibration control techniques. In a recent study, Azeloglu et al. [16, 17] have developed a mathematical model for container cranes and verified their numerical findings experimentally. They used earthquake loadings by using a shake table to measure the response of the flexible structure. Their results have exhibited good agreements between the experiments and the numerical predictions. Nevertheless, due to the complex dynamics of flexible structures, there could be inevitable modeling errors which can lead to poor performance of the control process or even to an instability of the flexible structure in several cases. In addition, measurement errors along with unpredictable actuator dynamics can further exaggerate the inaccurate modeling errors, leading to insufficient performance of the controller and consequently to undesired structural oscillations. In many cases, measurement data needs to undergo filtration procedures using software packages [18-22]. As a result, active vibration control can be much more challenging on the experiment site as compared to numerical simulations. This is mainly due to the complex measurement and control processes that involves unavoidable measurement inaccuracies and noise, nonlinearities, and modelling errors.

Due to simplicity and practicality of passive vibration control, passive elements such as vibration absorbers, vibration dampers are commonly used in mechanical, civil, aerospace, and many other industries. Vibration absorbers today can still provide adequate solutions to many engineering needs in vibration control area. Therefore, many researchers worked on the absorber design and their characteristics. Many design approaches and application areas have been proposed and presented in the literature for vibration absorbers. Sun et al. [23] investigated the application of dynamic vibration absorbers for the vibration control of multiple frequency harmonic excitations and compared the results with state-switched absorber. Bonello et al. [24] have designed an adaptive tuned vibration absorber (ATVA) with a smart variable stiffness element. Their design aimed the capability of retuning itself in response to a time-varying excitation frequency for effective vibration control over a range of frequencies. They investigated three different designs: (i) a pinned-pinned arch beam with fixed profile of slight curvature and variable preload through an adjustable natural curvature; (ii) a vibration absorber with a stiffness element formed from curved beams of adjustable curvature in parallel vibrating longitudinally; (iii) a vibration absorber with a variable geometry linkage as the stiffness element. Tuned mass absorbers are typically used to introduce damping in specific vibration modes of flexible structures with distributed mass. Krenk and Høgsberg [25] have proposed an explicit design method based on the equivalent absorber parameters and the structure represented by the relevant mode shape and the associated modal mass and stiffness, plus an additional quasi-static stiffness associated with the remaining modes. In 


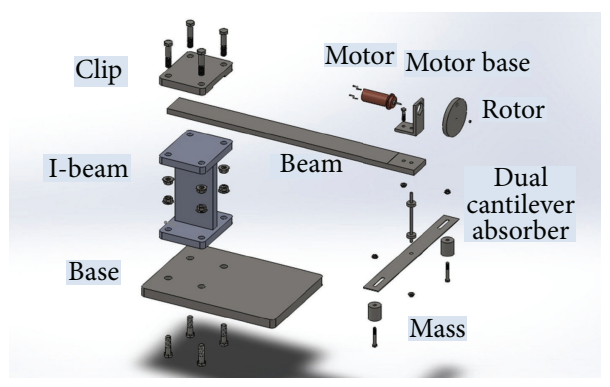

FIgURE 1: Exploded 3D view of the experimental setup design.

this study it was aimed to obtain fully balanced frequency curve for the dynamic amplification of the structural response and effective vibration control over a range of frequencies. Wang and Lo [26] proposed a novel hybrid dynamic balancer and vibration absorber that is cheaper than active dampers and more effective than passive dampers. Their design was capable of self-adjustment for the optimal damping location in order to optimize damping effects. Their experiments verified the efficacy of the proposed device in improving damping performance. Mashayekhi and Vahdati [27] have proposed a new fluid mount design in combination with a tuned vibration absorber and it was shown that an addition of a tuned vibration absorber to the fluid mount resulted in two notch frequencies which in turn may result in better cabin noise and vibration isolation. And the location of the new notch was easily controllable and directly dependent on the tuned vibration absorber's natural frequency with their design.

In this study, we have designed and implemented a beamtype twin dynamic vibration absorber for a cantilevered flexible structure under the influence of sinusoidal forcing at its tip. The sinusoidal forcing in this study is caused by an unbalanced rotor-motor assembly. The proposed design in the following section is convertible to a servo controlled, self-optimizing absorber system. Experiments and numerical studies have been conducted for comparison and effectiveness of the proposed absorber system. Numerical and experimental results demonstrated an adequate agreement and the proposed absorber was capable of eliminating the resonance. Next, the design, implementation, and results will be illustrated with case studies along with performance enhancements.

\section{Experimental Setup}

Most of the commercial laboratory test beds are designed as simply supported beam structures. However, cantilevered structures are frequently used as well in many engineering fields, especially in civil and mechanical engineering applications. In this research, we have designed and developed a test bed for vibration experiments with cantilevered structure in an attempt to control the rotor excited oscillations with an attached beam like dual-mass symmetrical absorber. In this work, design alternatives have been evaluated and a setup has been designed considering the performance, cost, weight/size, safety, and other properties. The design consists of a base which carries the setup, an I-beam to which the cantilevered beam is assembled, a motor with unbalanced rotor to excite the system, and speed control unit to adjust the rotor speed. Our results show that the platform has a distinct fundamental frequency and the designed absorber is capable of eliminating the vibrations at this resonance frequency. The developed modular model can provide a good source for vibration tests for a variety of control procedures as well.

Schematic of experimental setup and the photographic view of the fabricated laboratory prototype (which is currently under further developments) are given in Figures 1 and 2 , respectively. The primary structure for the experimental setup mainly consists of a cantilevered flexible beam, an electrical motor, an absorber (also in Figure 3), and mass unbalanced rotor attached to the shaft of a motor (also in Figure 4). The primary flexible structure is attached to a rigid I-beam structure which can be rigidly assembled to the base table. Underneath the motor assembly, the dual-mass, flexible beam-type vibration absorber is fixed to the primary structure. The motor is connected to a speed control unit through which the speed of rotation for the unbalanced rotor can be controlled. In order to measure the vibrations, we used an accelerometer and a vibrometer devices. Data acquisition has been realized with the help of Labview software.

\section{Experimental Observations and Numerical Investigations with Finite Element Analysis}

Theoretical investigations through modeling the cantilever beam as a distributed parameter dynamic system can be complex due to coupling and nonlinearities involved in the system, which may also require several unavoidable assumptions. Therefore, there are very few studies which models the primary and/or the absorber system as a continuous model for complicated models. Cavacece and Vita [28] have investigated double-ended cantilever beam as a distributed parameter dynamic vibration absorber. However, in their study the primary system has been considered simply as a single degree-of-freedom system which is subjected to harmonic forces and the absorber has been assumed as a cantilever beam without tip masses. They used Timoshenko bending theory and compared their results with results from Euler-Bernoulli model by Jacquot and Foster [29]. Although there were some discrepancies, their results were fairly in 


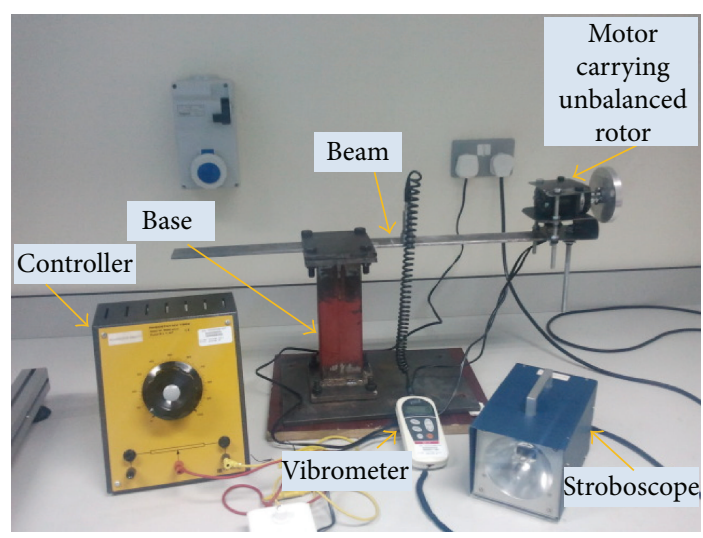

(a)

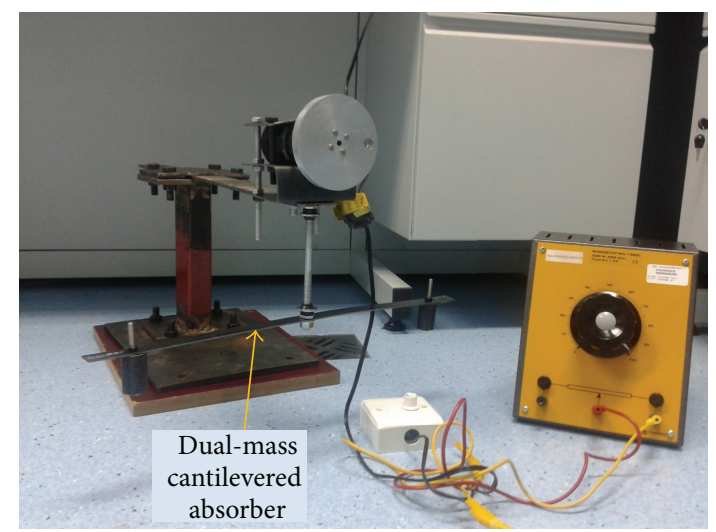

(b)

FIgURE 2: Photographs of the setup: (a) without absorber, (b) with the absorber.

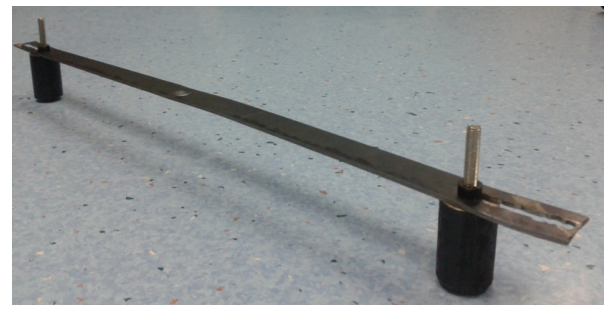

FIGURE 3: The cantilevered beam absorber with adjustable twin masses.
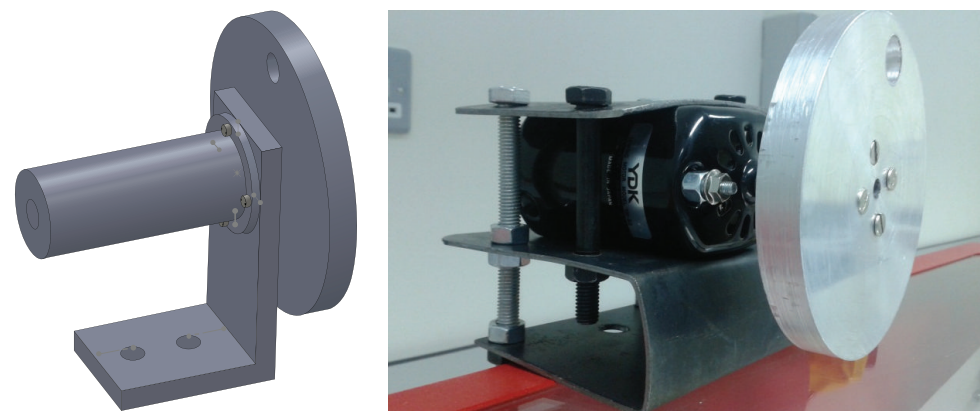

FIgURE 4: The unbalanced rotor-motor assembly.

agreement in both cases and they have proposed optimal parameters such as tuning ratio and mass ratio for the design engineer. In many cases, with distributed parameter systems, experimental verifications might be crucial. In this work, we have investigated a distributed parameter system experimentally, which can be difficult to analyze with analytical methods under various unavoidable assumptions, and verified the outcome with finite element analysis using the ANSYS software package. This sections are organized to first show the experimental observations followed by the finite element analysis results.

Although cantilevered structures exhibit different dynamic characteristics than lumped parameter systems, equivalent models can be developed to principally predict the fundamental natural frequency and general response of the structures. In this study, initial parameter selections have been organized using equivalent lumped models. For a lumped two degree-of-freedom system, equations of motion can be written in matrix form as $[1,2]$

$$
\begin{aligned}
& {\left[\begin{array}{cc}
m & 0 \\
0 & m_{a}
\end{array}\right]\left[\begin{array}{c}
\ddot{x} \\
\ddot{x}_{a}
\end{array}\right]+\left[\begin{array}{cc}
k+k_{a} & -k_{a} \\
-k_{a} & k_{a}
\end{array}\right]\left[\begin{array}{l}
x \\
x_{a}
\end{array}\right]} \\
& \quad=\left[\begin{array}{c}
F_{0} \sin \left(\omega_{\mathrm{dr}} t\right) \\
0
\end{array}\right],
\end{aligned}
$$

where $m$ and $x$ represent the primary system mass and displacement and $m_{a}$ and $k_{a}$ represent the absorber mass and displacement, respectively. $F_{0}$ is the amplitude of the harmonic force and $\omega_{\mathrm{dr}}$ represents the driving frequency. After solving these equations and some manipulations, the 


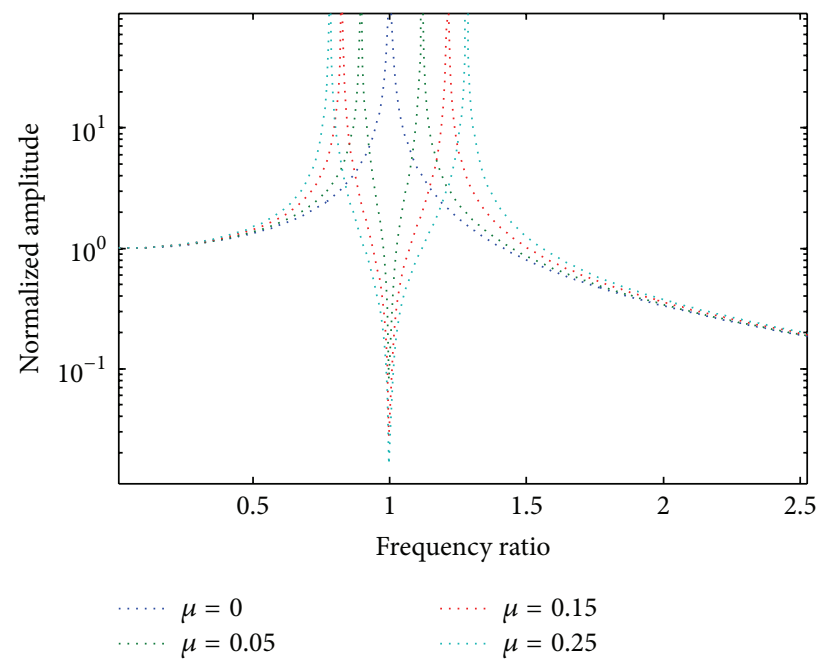

FIGURE 5: Normalized magnitude of primary mass versus normalized driving frequency.

magnitude of vibrations of the steady state motion of primary system $(X)$ and the absorber mass $\left(X_{a}\right)$ can be obtained as

$$
\begin{aligned}
X & =\frac{\left(k_{a}-m \omega_{\mathrm{dr}}^{2}\right) F_{0}}{\left(k+k_{a}-m \omega_{\mathrm{dr}}^{2}\right)\left(k_{a}-m \omega_{\mathrm{dr}}^{2}\right)-k_{a}^{2}}, \\
X_{a} & =\frac{k_{a} F_{0}}{\left(k+k_{a}-m \omega_{\mathrm{dr}}^{2}\right)\left(k_{a}-m \omega_{\mathrm{dr}}^{2}\right)-k_{a}^{2}}
\end{aligned}
$$

and the magnitude of $m_{a}$ and $k_{a}$ can be chosen such that the value $X$ in (2) is equal to zero.

With $\beta=\omega_{\mathrm{dr}} / \omega_{a}, f=\omega_{a} / \omega_{p}$, and $\mu=m_{a} / m$ the equation for the primary system can be rewritten as

$$
\frac{X k}{F_{0}}=\frac{1-\beta^{2}}{\left[1+\mu f^{2}-(\beta f)^{2}\right]\left[1-\beta^{2}\right]-\mu(f)^{2}} .
$$

In tuning case, $w_{a}=w_{p}$ and $f=1$; therefore, (4) will simplify to

$$
\frac{X k}{F_{0}}=\frac{1-\beta^{2}}{\left[1+\mu-\beta^{2}\right]\left[1-\beta^{2}\right]-\mu} .
$$

The ratio of absorber mass to the primary mass $\mu=m_{a} / m$ is usually taken between 0.05 and 0.25 (i.e., $0.05 \leq \mu \leq 0.25$ ). Here, $\mu$ plays an important role to separate the two natural frequencies of the primary and absorber system as shown in Figure 5. However, large $\mu$ is considered as poor design and $\mu$ has been selected to be approximately 0.15 in our design criteria.

As mentioned earlier, by using equivalent models, it may be possible to estimate the system response with the lumped parameter system and initial parameters have been selected accordingly. For the cantilevered beam of the primary system, $5 \mathrm{~mm}$ thickness steel material is nominated with a length of $380 \mathrm{~mm}$ and width of the $50 \mathrm{~mm}$. The absorber beam has a thickness of $2 \mathrm{~mm}$, with a length $250 \mathrm{~mm}$ at each side and width of $32 \mathrm{~mm}$. Tip mass which includes the motor assembly is measured to be $2.19 \mathrm{~kg}$ and absorber mass at each side has a mass of $0.34 \mathrm{~kg}$.

With the chosen parameters, initial experiments have been conducted by exciting the structure with an initial impact at the tip for the primary system to obtain the system response and the fundamental frequency. Typically, time responses of the system have been obtained with the Labview program for five-second time intervals. For data acquisition, sampling frequency rate of $18 \mathrm{kHz}$ has been selected and found appropriate throughout the experiments. Subsequently, recorded data have been transferred to Matlab [30] environment to perform spectrum analyses with a custom coded program.

Early tests have been performed with only the primary system of cantilevered beam and motor assembly to find out the fundamental natural frequency of the structure. Time responses and frequency spectrums obtained from experimental data are given in Figure 6. The initial result in Figure 6 for the primary system confirms that the system has a distinct fundamental frequency of about $8 \mathrm{~Hz}$ at which the first resonance is expected.

Resonance growth tests have been also performed on the system by running the motor from rest to a prescribed speed of $1000 \mathrm{rpm}$ with steady increments as shown in Figure 7. During the experiments, violent vibrations have been observed at around $480 \mathrm{rev} / \mathrm{min}$ as anticipated.

In order to control the oscillations when the unbalanced rotor runs at a speed corresponding to this fundamental frequency, the proposed tunable, dual-mass, symmetrical cantilevered absorber has been attached to the primary flexible structure which is also a cantilevered slender beam design. Further tests with the attached dynamic vibration absorber have been conducted by tapping the structure appropriately to stimulate the natural frequencies and then measuring the response. In this case, with the absorber unit attached to the primary system, the system is anticipated to exhibit two clearly distinct natural frequencies along with the higher frequencies associated with the flexible beam structures. Once again, system response to an initial excitation has been obtained as shown in Figure 8.

Now the system exhibits more than one distinct natural frequency at which the new resonances will occur. On the other hand, as the frequency spectrums indicate, it can be anticipated that significant reductions in vibration amplitudes can be obtained at the natural frequency of the primary system which was about $8 \mathrm{~Hz}$. These experimental results have been obtained with the stationary unbalanced rotor for 5 seconds time interval similar to the previous case.

Figure 9 shows the comparison between the frequency spectrums with and without absorber cases in a broader range. Higher frequencies inherent in the structure can be also noticed in the response plots clearly. It can be speculated that these higher frequencies may possibly be due to the cantilevered structures, or other components such as clamps and connection units. Absolutely, the excitation mode of the structure will also play an important role on the distinctiveness of the higher frequencies in the response tests and the results may differ from one test to another. Nevertheless, the comparison tests in Figure 10 will evidently 


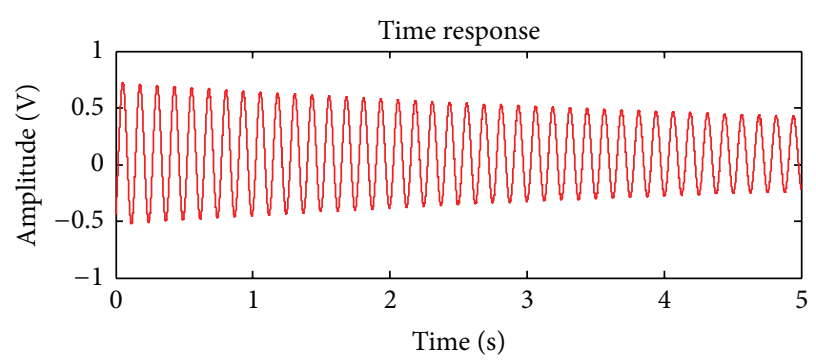

(a)

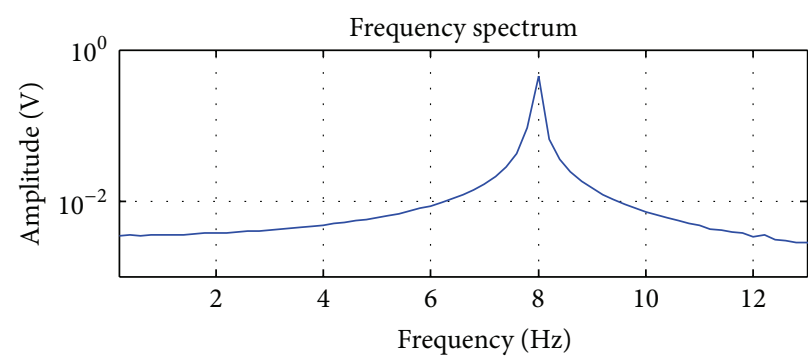

(b)

FIGURE 6: Transient response of the primary structure with a tip impact and without absorber. (a) Time history of the primary structure; (b) frequency spectrum of the response.

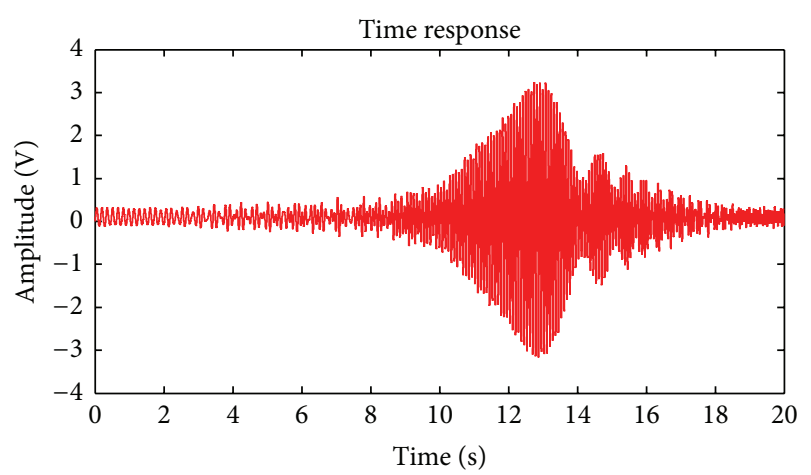

Figure 7: Resonance growth test.

show that significant vibration reductions can be attained at about $480 \mathrm{rpm}$ rotor speed which will be tested in the following experiment.

In order to confirm response plots with impact tests when the motor is at standstill, two experimental tests have been performed to observe the overall response of the flexible primary structure; one with absorber and the other without absorber while the unbalanced rotor runs at a constant speed in both cases. In both experimental analysis, the motor has been run at a constant speed of $480 \mathrm{rev} / \mathrm{min}$ continuously, which corresponds to primary system's fundamental natural frequency of about $8 \mathrm{~Hz}$. As can be seen equally from both time response and frequency spectrum plots in Figure 10, the proposed absorber practically eliminates the resonance and substantial reductions in vibration amplitudes are attainable. With extra fine tuning, the residual negligible amount of vibrations "with absorber" case could also be further reduced. Nevertheless, the measured amplitudes of the primary system without absorber have been observed at about 80 times of the oscillation amplitudes of the system with the absorber attachment. These experimental results demonstrate that the proposed beam-type twin dynamic vibration absorber is capable of significantly reducing the steady state oscillations for a cantilevered flexible structure carrying an unbalanced rotor.
Using similar parameters with the experimental tests, finite element model has been also developed in ANSYS and harmonic numerical analysis by finite element method (FEM) is performed in this section. 3D model is exploited to verify the earlier results for natural frequencies. Initially, 3D model of cantilever beam and lump mass on tip with and without absorber are created. Subsequently, areas and volumes are meshed with element PLANE182 and Solid 185, respectively, in ANSYS software (Figures 11(a)-11(d)). Spectrum of the response under broadband swept-harmonic excitation has been also obtained in Figure 11(e). Frequency responses from finite element analysis show that the amplitudes between two cases (with and without absorber) have similar pattern with the experiments although some differences are marked. Even though the excitation circumstances are somewhat dissimilar from the experiments, objectively comparable significant reductions in vibration amplitudes are apparent here as well.

\section{Conclusions}

In this study, we have designed and implemented a laboratory prototype of a cantilevered flexible structure and tested a beam-type twin dynamic vibration absorber to control undesired oscillations under the influence of sinusoidal forcing source at its tip. The sinusoidal forcing in this study is caused by an unbalanced rotor-motor assembly. A finite element model has been developed to investigate the proposed prototype numerically. Experiments and numerical studies have been conducted in conjunction for comparisons, and the effectiveness of the proposed absorber system is scrutinized in various operating conditions. Numerical and experimental results demonstrated an adequate agreement and the proposed dual-mass, cantilevered dynamic vibration absorber system was capable of significant vibration reductions on a flexible cantilevered platform carrying an unbalanced rotor system at its tip. The advantage of the proposed system comes from the fact that it can be practically tuned for variety of design and operating conditions and it is easy to apply variety of machines and structures. It can also be easily converted to a servo controlled self-optimizing absorber system. The 


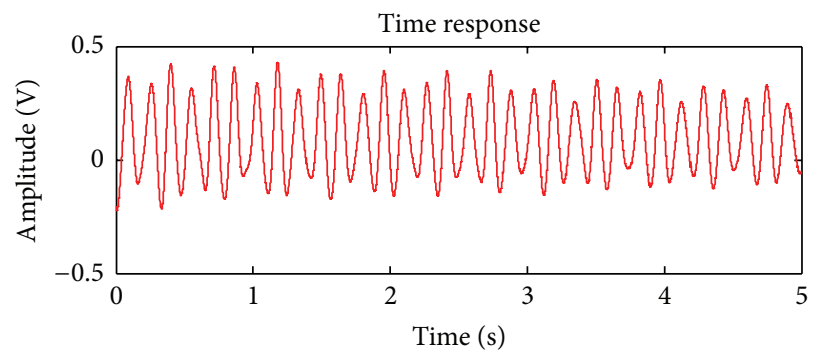

(a)

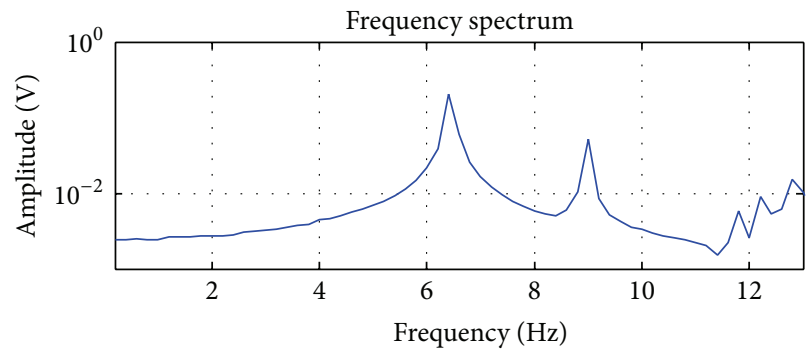

(b)

FIGURE 8: Transient response of the primary structure with a tip impact and with absorber. (a) Time history of the primary structure; (b) frequency spectrum of the response.

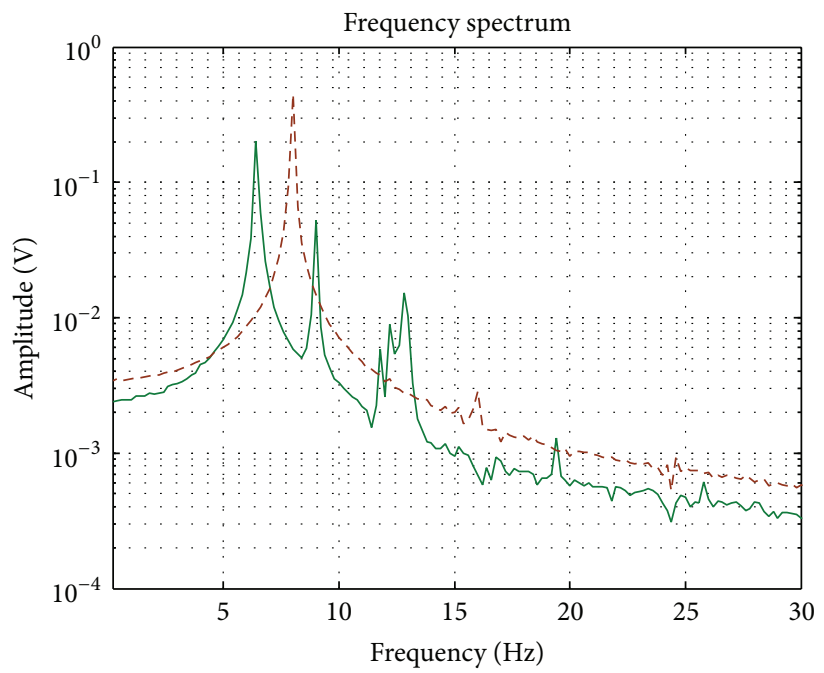

- With absorber

- - - Without absorber

FIGURE 9: Frequency spectrum of the response with and without absorber in a broader range of frequencies for comparison.

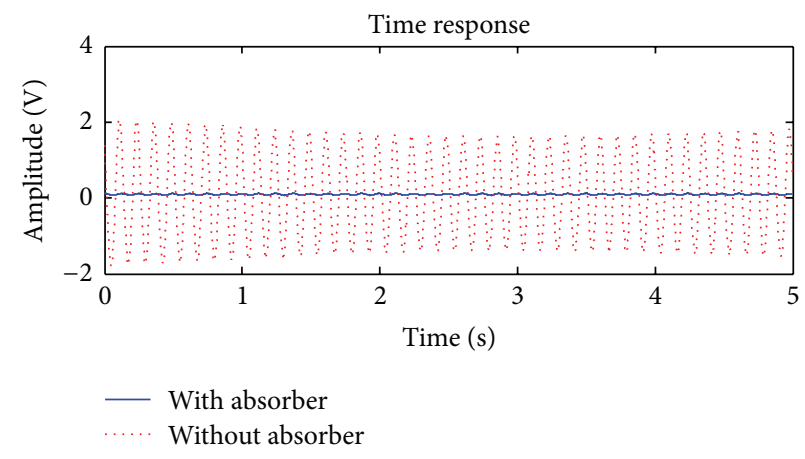

(a)

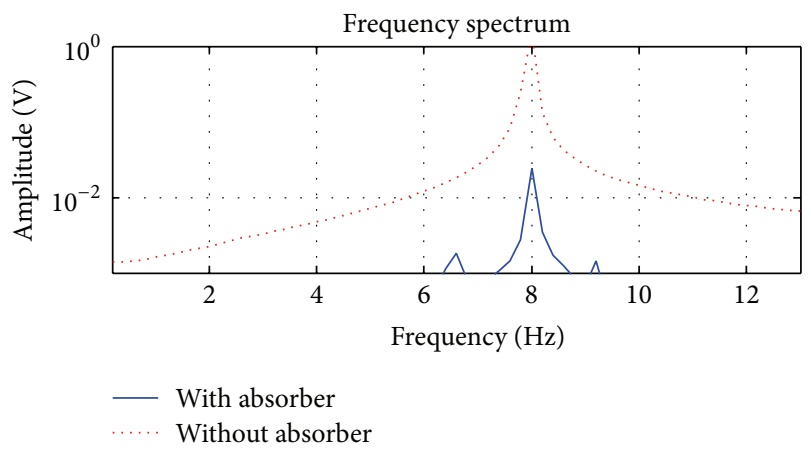

(b)

FIgURE 10: Experiments with and without absorber when the unbalanced rotor runs at $480 \mathrm{rpm}$. 


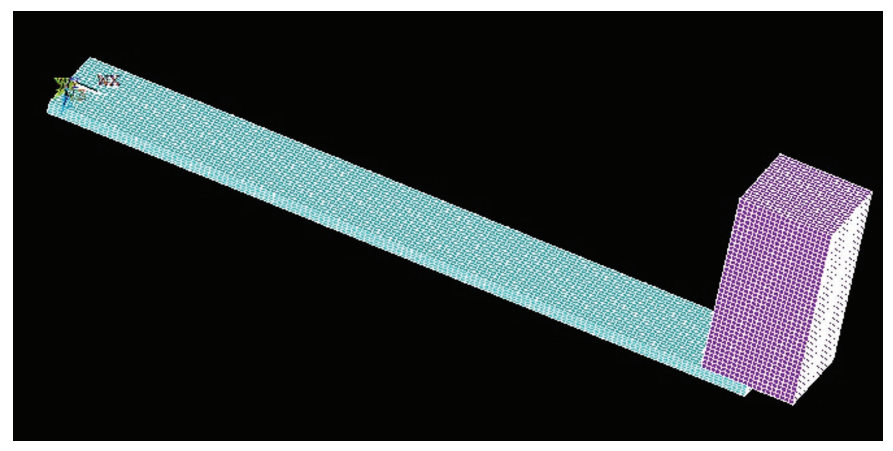

(a)

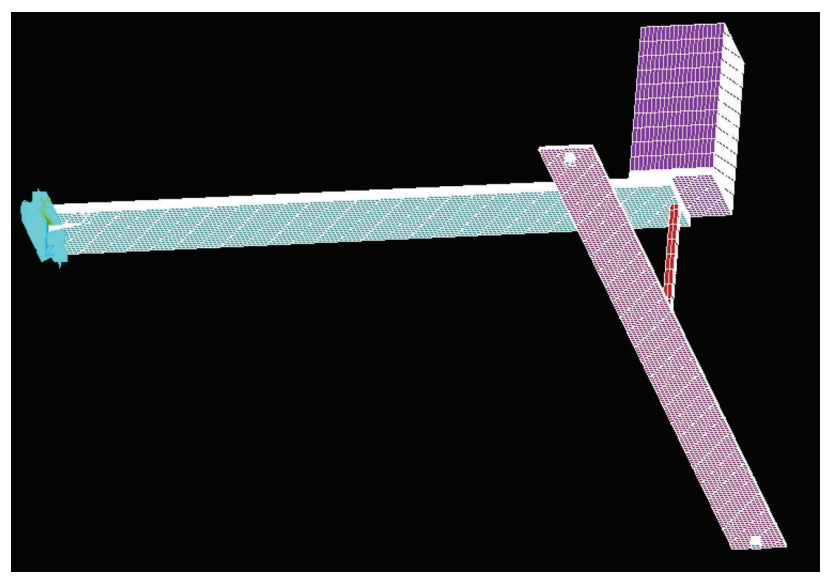

(c)

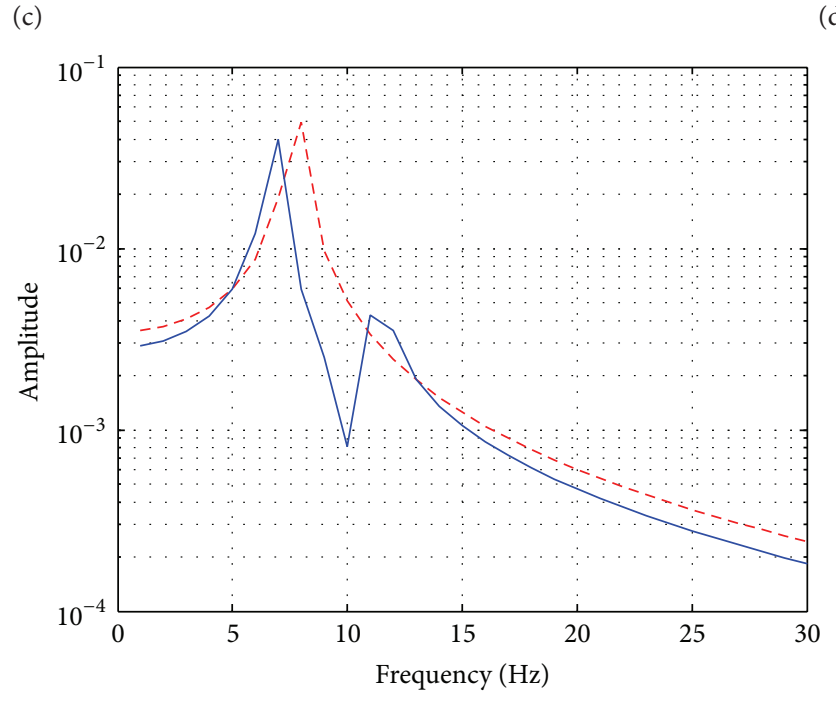

- - Without absorber

— With absorber

(e)

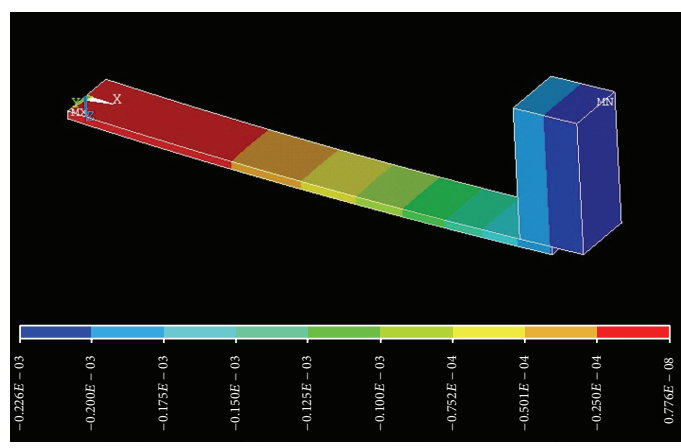

(b)

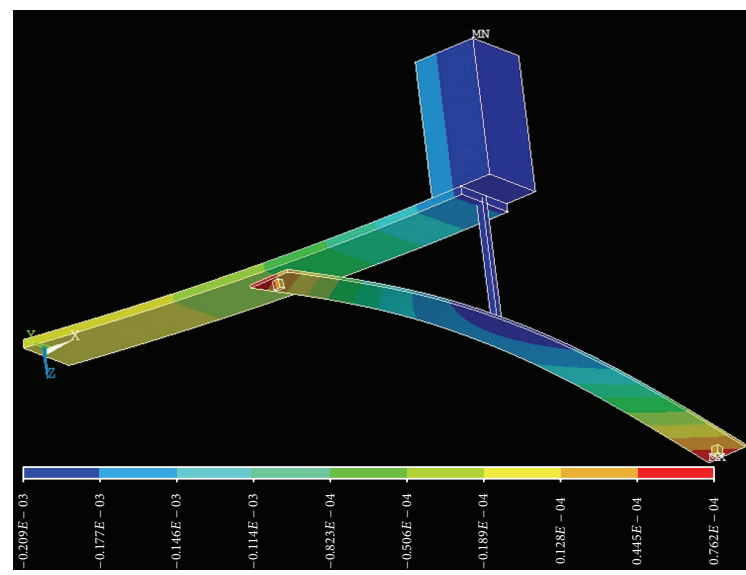

(d)

FIGURE 11: Harmonic analysis of the system: (a) modeling and meshing of the system without absorber, (b) normalized displacement in $z$ direction of the system without absorber, (c) modeling and meshing of the system with absorber, (d) normalized displacement in $z$ direction of the system with absorber, and (e) spectrum of the response under broadband swept-harmonic excitation. 
designed setup and the results in this paper can well serve for practicing engineers and future researchers. The considered system can be applied to a variety of flexible structures involving an unbalanced machine or under the influence of harmonic forcing.

\section{Conflict of Interests}

The authors declare that there is no conflict of interests regarding the publication of this paper.

\section{Acknowledgment}

This project has been principally funded by the equipment grant from Department of Mechanical \& Industrial Engineering, College of Engineering, Sultan Qaboos University, Muscat, Oman. The authors are grateful for the financial support obtained from this institution.

\section{References}

[1] W. T. Thomson, Theory of Vibrations with Applications, Prentice-Hall, Englewood Cliffs, NJ, USA, 1993.

[2] D. J. Inman, Engineering Vibration, Pearson Education, Essex, UK, 2014

[3] S. S. Rao, Mechanical Vibrations, Prentice-Hall, Upper Saddle River, NJ, USA, 2011.

[4] A. Özer and S. E. Semercigil, "An event-based vibration control for a two-link flexible robotic arm: numerical and experimental observations," Journal of Sound and Vibration, vol. 313, no. 3-5, pp. 375-394, 2008.

[5] Y. Okada, K. Matsuda, and J. Tani, "Active vibration control of a thin steel sheet," Shock and Vibration, vol. 2, no. 1, pp. 15-22, 1995.

[6] M. Biglar and H. R. Mirdamadi, "Integrated and consistent active control formulation and piezotransducer position optimization of plate structures considering spillover effects," Shock and Vibration, vol. 2014, Article ID 276714, 14 pages, 2014.

[7] M. Biglar, H. R. Mirdamadi, and M. Danesh, "Optimal locations and orientations of piezoelectric transducers on cylindrical shell based on gramians of contributed and undesired RayleighRitz modes using genetic algorithm," Journal of Sound and Vibration, vol. 333, no. 5, pp. 1224-1244, 2014.

[8] A. Özer, S. E. Semercigil, R. P. Kumar, and P. Yowat, "Delaying tool chatter in turning with a two-link robotic arm," Journal of Sound and Vibration, vol. 332, no. 6, pp. 1405-1417, 2013.

[9] A. Özer, A. Sekiguchi, and H. Arai, "Experimental implementation and analysis of robotic metal spinning with enhanced trajectory tracking algorithms," Robotics and Computer-Integrated Manufacturing, vol. 28, no. 4, pp. 539-550, 2012.

[10] A. Özer and S. E. Semercigil, "Effective vibration suppression of a maneuvering two-link flexible arm via an event-based stiffness controller," International Journal of Robotics and Automation, vol. 26, no. 2, pp. 171-184, 2011.

[11] L. Zhao and Z. D. Hu, "Active vibration control of an axially translating robot arm with rotating-prismatic joint using selfsensing actuator," Shock and Vibration, vol. 2015, Article ID 964139, 11 pages, 2015.

[12] X. Yalan, C. Jianjun, and W. Xiaobing, "Robust vibration control of uncertain flexible structures with poles placement," in Proceedings of the 1st International Symposium on Systems and
Control in Aerospace and Astronautics (ISSCAA '06), pp. 350354, January 2006.

[13] W.-H. Jee and C.-W. Lee, " $\mathrm{H}_{\infty}$ robust control of flexible manipulator vibration by using a piezoelectric-type servo-damper," Control Engineering Practice, vol. 2, no. 3, pp. 421-430, 1994.

[14] Y.-R. Hu and A. Ng, "Active robust vibration control of flexible structures," Journal of Sound and Vibration, vol. 288, no. 1-2, pp. 43-56, 2005.

[15] A. Montazeri, J. Poshtan, and A. Choobdar, "Performance and robust stability trade-off in minimax LQG control of vibrations in flexible structures," Engineering Structures, vol. 31, no. 10, pp. 2407-2413, 2009.

[16] C. O. Azeloglu and A. Edincliler, "Investigation of seismic behavior of container crane structures by shake table tests and mathematical modeling," Shock and Vibration, vol. 2014, Article ID 682647, 9 pages, 2014.

[17] C. O. Azeloglu, A. Sagirli, and A. Edincliler, "Mathematical modelling of the container cranes under seismic loading and proving by shake table," Nonlinear Dynamics, vol. 73, no. 1-2, pp. 143-154, 2013.

[18] N. Yagiz, "Vibration control of a building with ATMD under earthquake excitation," International Journal of Applied Mechanics and Engineering, vol. 8, no. 1, pp. 117-123, 2003.

[19] R. Guclu and H. Yazici, "Fuzzy logic control of a non-linear structural system against earthquake induced vibration," Journal of Vibration and Control, vol. 13, no. 11, pp. 1535-1551, 2007.

[20] R. Guclu and H. Yazici, "Vibration control of a structure with ATMD against earthquake using fuzzy logic controllers," Journal of Sound and Vibration, vol. 318, no. 1-2, pp. 36-49, 2008.

[21] R. Guclu and H. Yazici, "Seismic-vibration mitigation of a nonlinear structural system with an ATMD through a fuzzy PID controller," Nonlinear Dynamics, vol. 58, no. 3, pp. 553-564, 2009.

[22] R. Guclu and H. Yazici, "Self-tuning fuzzy logic control of a non-linear structural system with ATMD against earthquake," Nonlinear Dynamics, vol. 56, no. 3, pp. 199-211, 2009.

[23] H. L. Sun, P. Q. Zhang, H. B. Chen, K. Zhang, and X. L. Gong, "Application of dynamic vibration absorbers in structural vibration control under multi-frequency harmonic excitations," Applied Acoustics, vol. 69, no. 12, pp. 1361-1367, 2008.

[24] P. Bonello, M. J. Brennan, S. J. Elliott, J. F. V. Vincent, and G. Jeronimidis, "Designs for an adaptive tuned vibration absorber with variable shape stiffness element," Proceedings of the Royal Society A: Mathematical, Physical and Engineering Sciences, vol. 461, no. 2064, pp. 3955-3976, 2005.

[25] S. Krenk and J. Høgsberg, "Tuned mass absorber on a flexible structure," Journal of Sound and Vibration, vol. 333, no. 6, pp. 1577-1595, 2014.

[26] Y. R. Wang and C. Y. Lo, "Design of hybrid dynamic balancer and vibration absorber," Shock and Vibration, vol. 2014, Article ID 397584, 18 pages, 2014.

[27] M. J. Mashayekhi and N. Vahdati, "Application of tuned vibration absorbers in fluid mounts," Shock and Vibration, vol. 16, no. 6, pp. 565-580, 2009.

[28] M. Cavacece and L. Vita, "Optimal cantilever dynamic vibration absorbers by timoshenko beam theory," Shock and Vibration, vol. 11, no. 3-4, pp. 199-207, 2004.

[29] R. G. Jacquot and J. E. Foster, "Optimal cantilever dynamic vibration absorbers," Journal of Engineering for Industry, Transactions of the ASME, vol. 99, no. 1, pp. 138-141, 1977.

[30] Matlab, The Language of Technical Computing, Using MATLAB Version 5, The MathWorks, Natick, Mass, USA, 1997. 

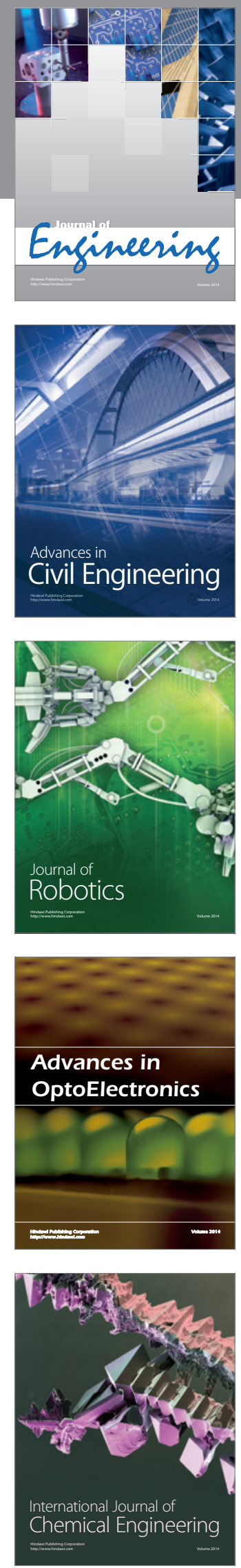

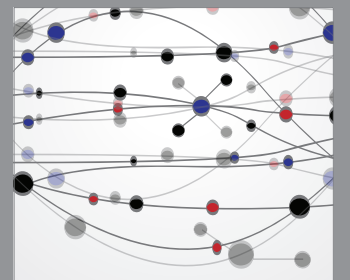

The Scientific World Journal
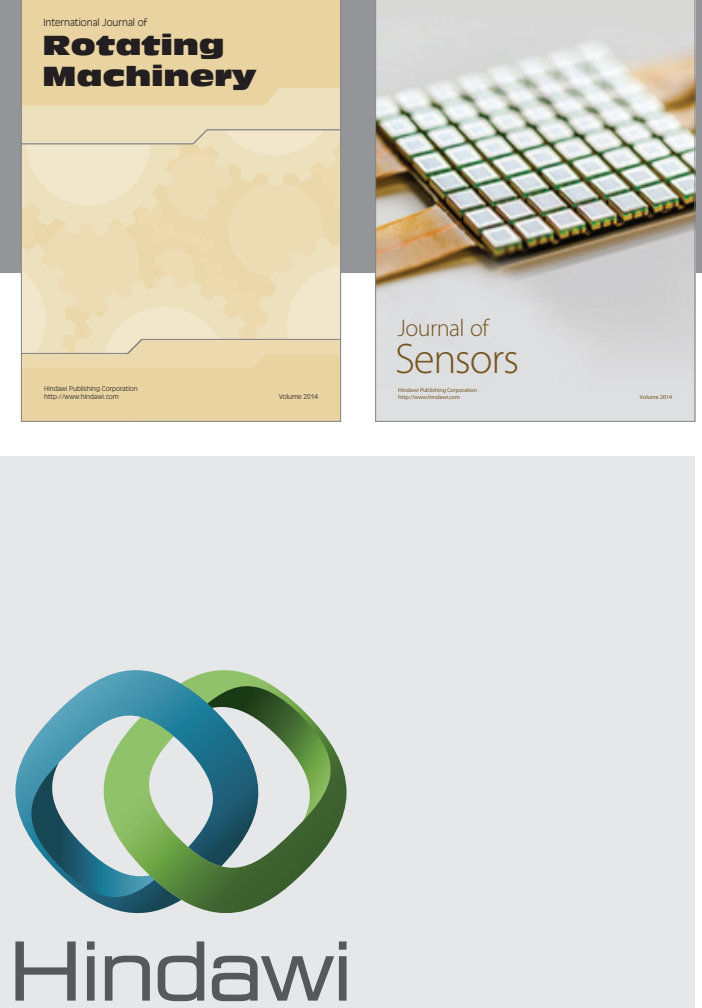

Submit your manuscripts at http://www.hindawi.com
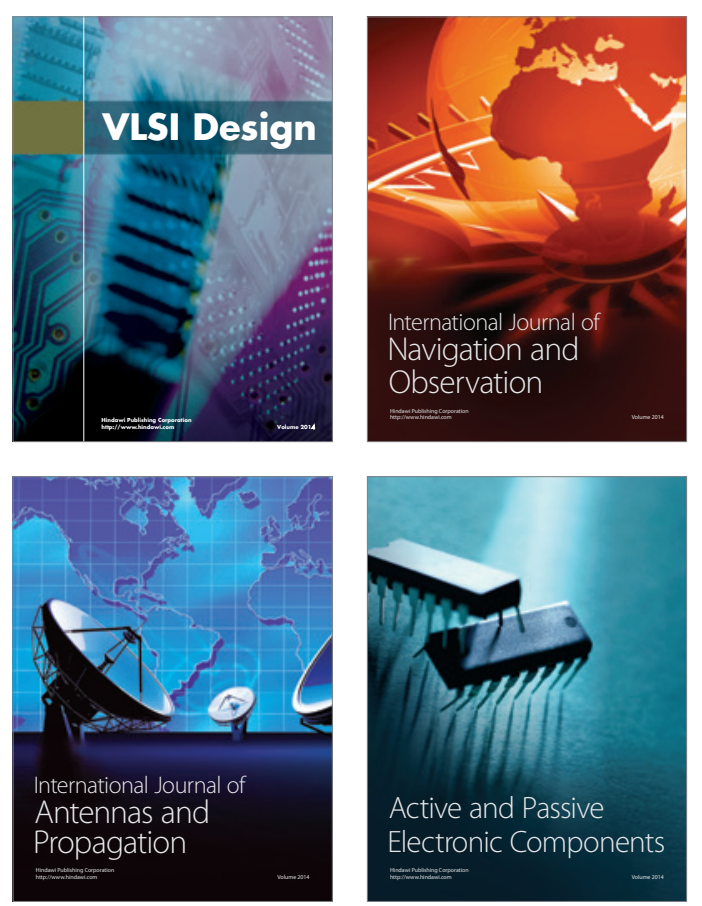
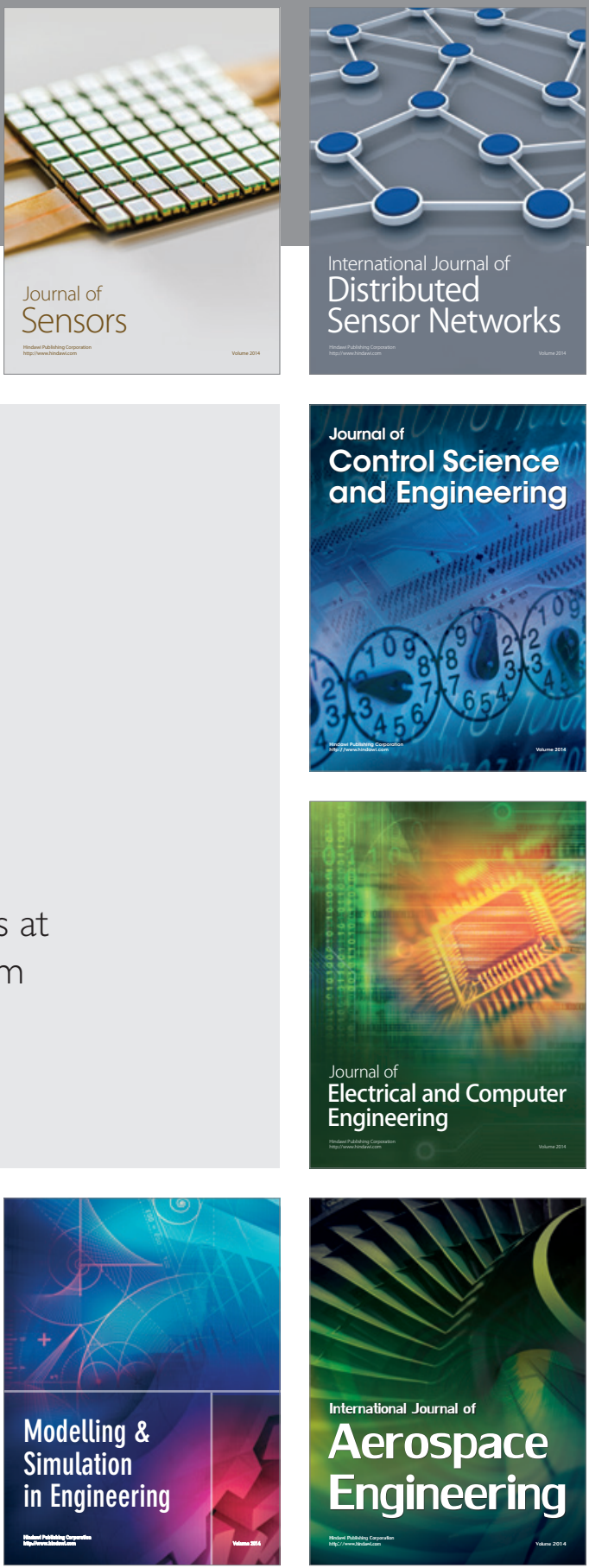

Journal of

Control Science

and Engineering
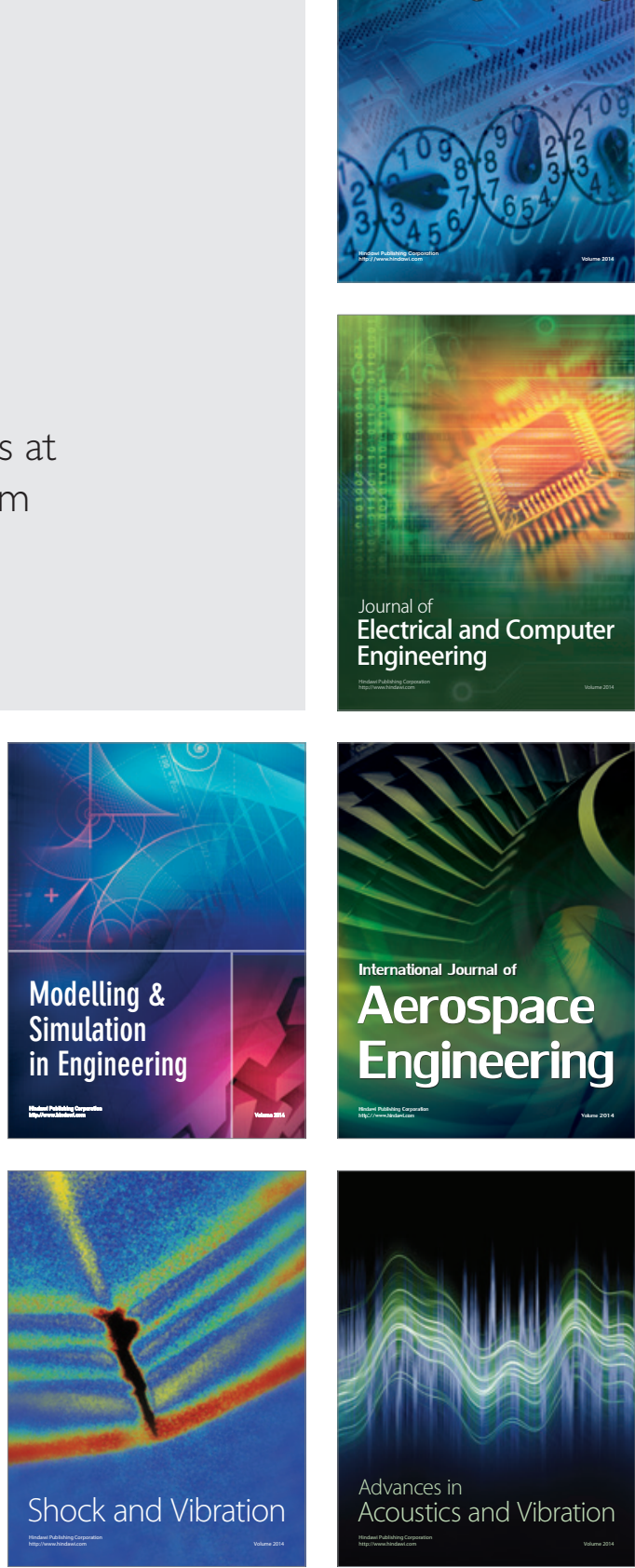